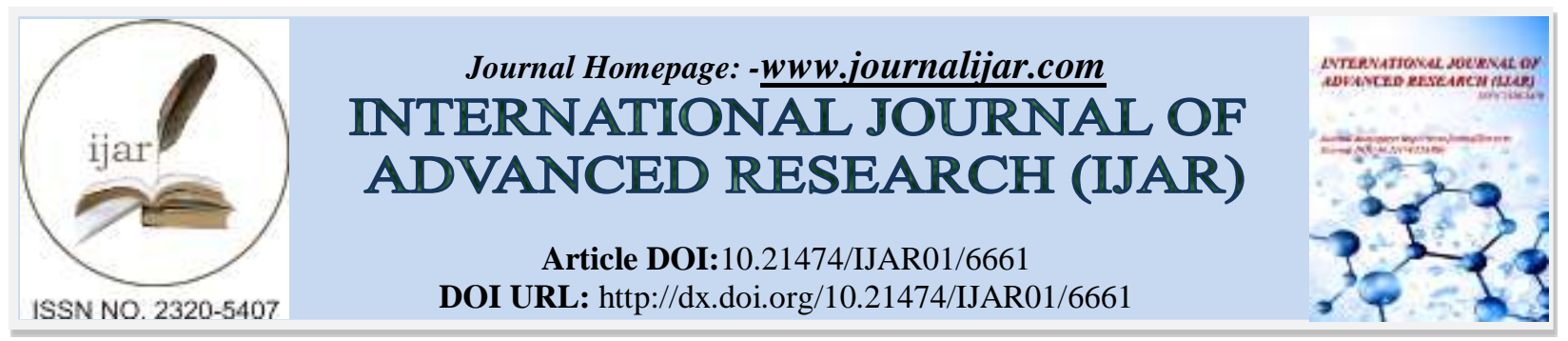

RESEARCH ARTICLE

\title{
STUDY OF EFFECT OF PLACEMENT OF GROYNE ON SHARP BEND IN RIVER USING CFD ANALYSIS.
}

Kirti Singh.

Post graduate student, Department of Civil Engineering, Delhi Technological University, Bawana road, Delhi, India.

\section{Manuscript Info}

Manuscript History

Received: 04 January 2018

Final Accepted: 06 February 2018

Published: March 2018

Keywords:-

Groyne, angle of installation, separation, Ansys Fluent.

\begin{abstract}
In a meandering river the most basic segment of the river is the point at which it changes its curvature, at this segment complex flow happens, also the additional forces like centrifugal force that become possibly the most important factor because of the curvature make the bank protection against scouring essential. As there are not much examination that focus on the investigation of flow in a channel bend, inferable from the complexities. Reenacting the shape and waterway conditions in a flume is likewise intense, fortunately making utilization of computational dynamic programming help in simulation studies. Groynes are usually installed along the river side and those are used for a lot of application such as bank protection. These structures would change the flow pattern and prevent bank erosion. So understanding turbulent flow pattern around groynes for designing groyne installation criteria is very important. In this subcritical flow pattern around an indirect groyne in different angle of installation was established and 3D simulations in Fluent software have been applied to investigate the effect of angle of groyne installation on separation zone length behind it. In this examination groyne at various positions on the channel bend are investigated utilizing ANSYS Fluent software. The turbulence model used was standard k-epsilon model for the flow. In all cases the channel width and inlet flow depth were constant and the bed slope of the channel was zero. The tip velocities, protected length and bed shear stress were compared for different positions of groyne. The most effective position of the groyne among the different cases was suggested in this study, also suggestions were made for the appropriate length of groyne.
\end{abstract}

Copy Right, IJAR, 2018,. All rights reserved.

\section{Introduction:-}

In natural channels and bodies of water the bed is not fixed but is composed of mobile particles; e.g. gravel, sand or silt. These may be dislodged and moved by the flow - the process of sediment transport. These sediments travel from the river to various reservoirs which reduce the storage capacity of the reservoir, reduces the navigation depth, and changes the path of river. This makes the river to meander.

The protection of banks of a meandering river is done by providing structures like groynes on the concave face of the channel. They are usually provided in series for longer channel and singly for curved sections. 
Because of the wide applications of these structures in river and coastal engineering, in addition to the numerical and experimental studies in the past, many researches have been conducted in recent years, such as experiments by Ettema, R., Muste, M. (2004). They presented the flow-thalweg alignment around a dike, and area extent of the wake immediately downstream of a dike. The experiments show that use of a shear-stress parameter as the primary criterion for dynamic similitude influences these features. In 2005, Uijttewaal studied the flow pattern around the groynes with different shapes experimentally. He aimed at finding efficient alternative designs (various groyne shapes), in the physical, economical, and ecological sense, for the standard groynes in the large rivers of Europe. According to their observation, it was demonstrated that the turbulence properties around the groyne can be manipulated by changing the permeability and slope of the groyne head. It was also shown that for submerged conditions the flow becomes more complex and locally dominated by three-dimensional effects. Also, Yeo et al. performed some experiments (69 experiments) to produce a design guideline in Korea and to examine the downstream separation region of a groyne under various groyne lengths and installation angles with various degrees of groyne permeability. Prasad Krishna S, Indulekha K.P, Balan.K, (2015) analyzed the effects of placing of groynes at different angles from $45^{\circ}$ to $135^{\circ} \&$ found the most effective arrangement for minimizing the erosion of bank. J.Yazdi \& H. Sarkardeh(1989), simulated the flow patterns around a single spur dike with free surface flow using a numerical model known as fluent. The reattachment length for various conditions was measured using numerical results, flow patterns and bed-shear stress distribution was presented for repelling, attracting \& vertical spurs together.

The k- $\omega$ turbulence model with the VOF method was used to simulate fully 3D flows. Shamloo Hamid and Pirzadeh Bahareh, established the subcritical flow pattern around an indirect groyne in six different angle of installation and 3D simulations in Fluent software have been applied to investigate the effect of angle of groyne installation on separation zone length behind it. Also, Majid Fazli, Masoud Ghodsian, and Seyed Ali Akbar Salehi Neyshabouri, (2008), presented the experiments on flow field and scour around a spur dike in a $90^{\circ}$ channel bend. Experiments were conducted for different lengths and different locations of spur dikes at the bend with different values of discharge. The 3-D flow fields around a spur dike were investigated. The maximum depth of scour was correlated to the Froude numbers, lengths and the locations of spur dike in the bend.

\section{Numerical Model Description:-}

FLUENT is the CFD solver for complex flow ranging from incompressible to highly compressible flows. It provides multiple choices of solver option, combined with a convergence-enhancing multi-grid method. Because of its optimum solution efficiency and accuracy, it has been selected to simulate the groyne in this research. The FLUENT's parallel solver has the ability to use multiple processors that may be executing on the same computer, or on different computers in a network to decrease the computing time. This capability has been used in the present study using a dual core computer in order to reduce the simulation time and numerical efforts. Conservative form of the Navier-Stokes equations using the Finite Volume method on structured Cartesian coordinate grid system applied to solve the flow parameters. Reynolds Stress turbulence model (RSM) in Fluent software, applied to estimate the turbulent flow field. The details of governing equations and RSM model can be found in FLUENT user's guide.

The simulated channel section is designed with criteria of Mean Radius/Width=2.

The channel width is assumed a constant value of $20 \mathrm{~m}$, thus the mean radius is taken as $40 \mathrm{~m}$. The height of the channel section is taken as $8 \mathrm{~m}$.

The numerical simulations were taken for groyne of 6 different sizes, that is, $4 \mathrm{~m} ; 3.5 \mathrm{~m} ; 3 \mathrm{~m}$ in length and $0.2 \& 0.5 \mathrm{~m}$ width. These sizes conform to the design criteria giving by the 'Indian Standard Code IS 8408:1994', which restricts the size of groyne to $1 / 5$ times the channel width.

Three different velocities of $2 \mathrm{~m} / \mathrm{s} ; 1.5 \mathrm{~m} / \mathrm{s}$ and $1 \mathrm{~m} / \mathrm{s}$ were taken for the numerical simulations to study the variation of velocity flow patterns and shear stress. The primary aim of this project was to protect the concave face of the river bank and find the most efficient placement of the groyne in the bend channel.

\section{Conservation Equations:-}

1. Conservation of Mass

$$
\frac{\partial \rho}{\partial \tau}+\frac{\partial \rho U_{i}}{\partial x_{i}}=0
$$


2. Conservation of momentum

3. Continuity equation

$$
\frac{\partial \rho U_{i}}{\partial \tau}+\frac{\partial \rho U_{j} U_{i}}{\partial x_{j}}=\frac{\partial}{\partial x_{j}}\left(\mu \frac{\partial U_{i}}{\partial x_{j}}-\rho u_{i} u_{j}\right)-\frac{\partial P}{\partial x_{i}}+S_{u i}
$$

4. Energy equation

$$
\frac{\partial \rho}{\partial t}+\nabla \cdot(\rho V)=0
$$

$$
\begin{aligned}
& \rho \frac{D}{D t}\left(e+\frac{V^{2}}{2}\right)=\rho q+\frac{\partial}{\partial x}\left(k \frac{\partial T}{\partial x}\right)+\frac{\partial}{\partial y}\left(k \frac{\partial T}{\partial y}\right)+\frac{\partial}{\partial z}\left(k \frac{\partial T}{\partial z}\right)-\frac{\partial(u p)}{\partial x}-\frac{\partial(v p)}{\partial y}-\frac{\partial(w p)}{\partial z}+\frac{\partial\left(u \tau_{x x}\right)}{\partial x}+\frac{\partial\left(u \tau_{y x}\right)}{\partial y}+ \\
& \frac{\partial\left(u \tau_{z x}\right)}{\partial z}+\frac{\partial\left(v \tau_{x y}\right)}{\partial x}+\frac{\partial\left(v \tau_{y y}\right)}{\partial y}+\frac{\partial\left(v \tau_{z y}\right)}{\partial z}+\frac{\partial\left(w \tau_{x z}\right)}{\partial x}+\frac{\partial\left(w \tau_{y z}\right)}{\partial y}+\frac{\partial\left(w \tau_{z z}\right)}{\partial z}+\rho f . V
\end{aligned}
$$

\section{Methods:-}

\section{Modelling groyne placement:-}

A modelling of groyne placement on the river bend based on current flow analysis having a goal to develop the good placement of groyne that were to be considered with the length of the river bank be protected. Simulation Studies were conducted on hypothetical channel for assessment of effect of placement of groyne on separation zone length behind it and consequent meandering of the banks of river.

The use of computational fluid dynamics software is very much helpful in solving partial differential equations based on conservation principles.

The main purpose of the simulation described below is to get the optimum number of observations for further analysis to be done. Though, the observations taken here are not exhaustive in any respect. As to find the best placement of the groyne, increase in the data would also increase the accuracy of the results. So, the properties considered for the purpose of simulations are as follows.

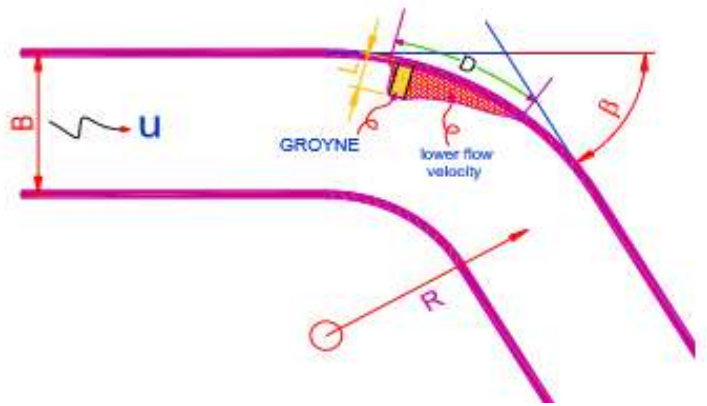

Fig.1:- Parameters that influence to the length of the river bank be protected.

Morphology Factor

B : River width

$\mathrm{R}$ : Radius bend

$\beta$ : Angle Bend

Hydrology and Hydrometry factor

$\mathrm{u}$ : Flow Velocities

h : Water depth

Groyne placement factor

$\mathrm{L}$ : Length of Groyne

$\beta$ : in this research $\beta=90^{\circ}$

\section{Case Research be purposed:-}

As previously described for a good placement of groyne on the river bend will be analysis based on the behavior of flow current that occurs. The purpose of Case research was to carried out flow simulation repeatedly on various cases of groyne placement with several alternative flows. There are eighteen case taken on the same angle of river bend i.e. $\beta=90^{\circ}$ and constant river width of $\mathrm{B}=20 \mathrm{~m}$ to be proposed. The research is done by 90 times simulation to 
the groyne placement on the river bend.

Three different velocities of $2 \mathrm{~m} / \mathrm{s} ; 1.5 \mathrm{~m} / \mathrm{s} ; 1 \mathrm{~m} / \mathrm{s}$ are used in this study for the results. These input velocities are average velocities through which the computations are initialized.

The lengths of groyne used for study are $4 \mathrm{~m}, 3.5 \mathrm{~m}$ and $3 \mathrm{~m}$. These groyne are non permeable in nature. The groyne used on the concave bank of channel is repelling in nature and thus they are pointed upstream.

Position of Groyne For Study:-

In this study, groyne placement was considered at three different sections along the channel bend. The position for the initial section for the placement was decided by taking into observation the flow velocities in a channel without any groyne that is without any protection.

Position A-at upstream arc of the bend

Position B-at $1 / 4^{\text {th }}$ upstream arc

Position C-at middle of the arc bend

The three variations of the position are as shown in Fig. 2,

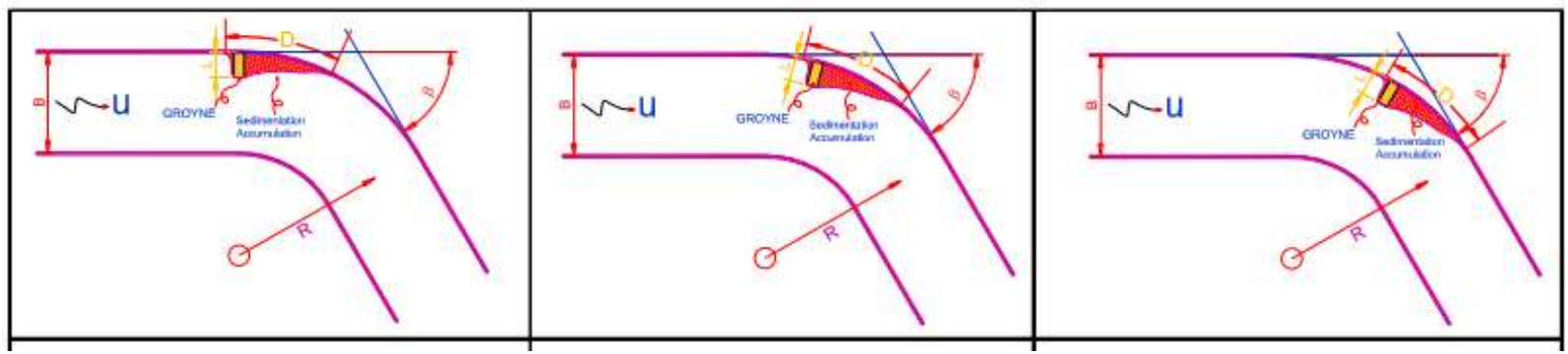

Fig. 2:-Three various groyne position placement on the river bend

Position A-Groyne at the upstream arc Position B-Groyne at the 1/4 upstream arc Position C-Groyne at the centre arc of the river bend

Below are the different positions of groyne placement at the concave face of the bend

Table 1:-Position of different groyne at concave face

\begin{tabular}{|l|l|l|l|l|}
\hline & $\mathrm{A}$ & $\mathrm{b}$ & $\mathrm{c}$ & $\mathrm{d}$ \\
\hline POSITION & $\theta=15^{\circ}$ & $\theta=30^{\circ}$ & $\theta=45^{\circ}$ & $\theta=60^{\circ}$ \\
\hline
\end{tabular}

Different cases were run for groyne of different size, position and approach velocity.

The following table contains the values of tip velocity, maximum velocity, maximum shear stress, initial shear stress and protected length obtained from the Fluent software.

Software Output:-

For position A

\begin{tabular}{|c|c|c|c|c|c|c|c|c|c|c|c|}
\hline GROYNE & $\begin{array}{l}\text { Length } \\
\text { of } \\
\text { groyne } \\
\text { (m) }\end{array}$ & $\begin{array}{l}\text { Width } \\
\text { of } \\
\text { groyne } \\
\text { (m) }\end{array}$ & $\begin{array}{l}\text { Approach } \\
\text { velocity } \\
\mathrm{V}_{\text {app }} \\
(\mathrm{m} / \mathrm{s})\end{array}$ & $\begin{array}{l}\text { Maximum } \\
\text { velocity } \\
\mathrm{V}_{\max } \\
(\mathrm{m} / \mathrm{s})\end{array}$ & $\begin{array}{l}\text { Tip } \\
\text { velocity } \\
\mathrm{V}_{\text {tip }} \\
(\mathrm{m} / \mathrm{s})\end{array}$ & $\begin{array}{l}\text { Max. } \\
\text { shear } \\
\text { stress, } \\
\Gamma_{\max } \\
(\mathrm{Pa})\end{array}$ & $\begin{array}{l}\text { Initial } \\
\text { shear } \\
\text { stress, } \\
\Gamma_{\mathrm{o}} \\
(\mathrm{Pa})\end{array}$ & $\begin{array}{l}\text { Protected } \\
\text { length, D } \\
\text { (m) }\end{array}$ & $\mathrm{V}_{\mathrm{tip}} / \mathrm{V}_{\mathrm{app}}$ & $\mathrm{D} / \mathrm{L}$ & $\mathrm{L} / \mathrm{B}$ \\
\hline $1 \mathrm{a}$ & 3 & 0.2 & 1 & 1.776 & 1.562 & 16.7104 & 1.259 & 65.63 & 1.562 & 21.876 & 0.15 \\
\hline $1 b$ & 3 & 0.5 & 1 & 1.771 & 1.228 & 16.7104 & 1.259 & 75.323 & 1.228 & 25.107 & 0.15 \\
\hline $1 \mathrm{c}$ & 3.5 & 0.2 & 1 & 1.904 & 1.262 & 19.117 & 1.259 & 70.514 & 1.262 & 20.1468 & 0.175 \\
\hline $1 d$ & 3.5 & 0.5 & 1 & 1.902 & 1.61 & 16.7104 & 1.259 & 73.748 & 1.61 & 21.0708 & 0.175 \\
\hline $1 \mathrm{e}$ & 4 & 0.2 & 1 & 2.009 & 1.27 & 16.7104 & 1.259 & 73.964 & 1.27 & 18.491 & 0.2 \\
\hline $1 \mathrm{f}$ & 4 & 0.5 & 1 & 2.017 & 1.57 & 16.7104 & 1.259 & 75.939 & 1.57 & 18.9847 & 0.2 \\
\hline $2 a$ & 3 & 0.2 & 1.5 & 2.656 & 1.37 & 16.7104 & 2.702 & 87.46 & 0.9133 & 29.1533 & 0.15 \\
\hline
\end{tabular}




\begin{tabular}{|l|l|l|l|l|l|l|l|l|l|l|l|}
\hline 2b & 3 & 0.5 & 1.5 & 2.734 & 0.612 & 16.7104 & 2.702 & 74.313 & 0.408 & 24.771 & 0.15 \\
\hline 2c & 3.5 & 0.2 & 1.5 & 2.897 & 0.3827 & 19.1117 & 2.702 & 72.468 & 0.2551 & 20.7051 & 0.175 \\
\hline 2d & 3.5 & 0.5 & 1.5 & 2.844 & 2.69 & 16.7104 & 2.702 & 78.12 & 1.7933 & 22.32 & 0.175 \\
\hline 2e & 4 & 0.2 & 1.5 & 3.021 & 0.355 & 16.7104 & 2.702 & 86.42 & 0.2366 & 21.605 & 0.2 \\
\hline 2f & 4 & 0.5 & 1.5 & 3.0228 & 1.539 & 16.7104 & 2.702 & 76.179 & 1.026 & 19.0447 & 0.2 \\
\hline 3a & 3 & 0.2 & 2 & 3.673 & 1.835 & 22.724 & 4.6429 & 78.92 & 0.9175 & 26.3066 & 0.15 \\
\hline 3b & 3 & 0.5 & 2 & 3.683 & 0.572 & 17.2105 & 4.6429 & 76.93 & 0.286 & 25.6433 & 0.15 \\
\hline 3c & 3.5 & 0.2 & 2 & 3.837 & 0.586 & 24.148 & 4.6429 & 75.75 & 0.293 & 21.6428 & 0.175 \\
\hline 3d & 3.5 & 0.5 & 2 & 3.872 & 2.481 & 18.508 & 4.6429 & 79.58 & 1.2405 & 22.7371 & 0.175 \\
\hline 3e & 4 & 0.2 & 2 & 4.021 & 0.736 & 25.447 & 4.6429 & 78.42 & 0.368 & 19.605 & 0.2 \\
\hline 3f & 4 & 0.5 & 2 & 3.975 & 1.515 & 20.0193 & 4.6429 & 69.38 & 0.7575 & 17.345 & 0.2 \\
\hline
\end{tabular}

For position $\mathrm{B}$

\begin{tabular}{|l|l|l|l|l|l|l|l|l|l|l|l|}
\hline GROYNE & $\begin{array}{l}\text { Length } \\
\text { of } \\
\text { groyne } \\
(\mathrm{m})\end{array}$ & $\begin{array}{l}\text { Width } \\
\text { of } \\
\text { groyne } \\
(\mathrm{m})\end{array}$ & $\begin{array}{l}\text { Approach } \\
\text { velocity } \\
\mathrm{V}_{\text {app }} \\
(\mathrm{m} / \mathrm{s})\end{array}$ & $\begin{array}{l}\text { Maximum } \\
\text { velocity } \\
\mathrm{V}_{\text {max }} \\
(\mathrm{m} / \mathrm{s})\end{array}$ & $\begin{array}{l}\text { Tip } \\
\text { velocity } \\
\mathrm{V}_{\text {tip }} \\
(\mathrm{m} / \mathrm{s})\end{array}$ & $\begin{array}{l}\text { Max. } \\
\text { shear } \\
\text { stress, } \\
\Gamma_{\max } \\
(\mathrm{Pa})\end{array}$ & $\begin{array}{l}\text { Initial } \\
\text { shear } \\
\text { stress, } \\
\Gamma_{\mathrm{o}} \\
(\mathrm{Pa})\end{array}$ & $\begin{array}{l}\text { Protected } \\
\text { length, D } \\
(\mathrm{m})\end{array}$ & $\mathrm{V}_{\text {tip }} / \mathrm{V}_{\text {app }}$ & D/L & \\
\hline 1a & 3 & 0.2 & 1 & 1.5021 & 1.0923 & 2.825 & 1.259 & 68.467 & 1.0923 & 22.8223 & 0.15 \\
\hline 1b & 3 & 0.5 & 1 & 1.494 & 0.1610 & 2.6953 & 1.259 & 68.83 & 0.161 & 22.943 & 0.15 \\
\hline 1c & 3.5 & 0.2 & 1 & 1.5833 & 1.083 & 3.106 & 1.259 & 68.786 & 1.083 & 19.6531 & 0.175 \\
\hline 1d & 3.5 & 0.5 & 1 & 1.6181 & 0.171 & 2.8237 & 1.259 & 68.68 & 0.171 & 19.6228 & 0.175 \\
\hline 1e & 4 & 0.2 & 1 & 1.6618 & 0.198 & 3.2845 & 1.259 & 68.72 & 0.198 & 17.18 & 0.2 \\
\hline 1f & 4 & 0.5 & 1 & 1.666 & 0.2342 & 3.0243 & 1.259 & 68.88 & 0.2342 & 17.22 & 0.2 \\
\hline 2a & 3 & 0.2 & 1.5 & 2.2739 & 1.2798 & 6.0847 & 2.702 & 68.637 & 0.8532 & 22.879 & 0.15 \\
\hline 2b & 3 & 0.5 & 1.5 & 2.2642 & 0.89 & 5.7094 & 2.702 & 68.703 & 0.5933 & 22.901 & 0.15 \\
\hline 2c & 3.5 & 0.2 & 1.5 & 2.4043 & 0.72 & 6.631 & 2.702 & 68.775 & 0.48 & 19.65 & 0.175 \\
\hline 2d & 3.5 & 0.5 & 1.5 & 2.4397 & 0.70 & 4.8593 & 2.702 & 68.8610 & 0.4666 & 19.6745 & 0.175 \\
\hline 2e & 4 & 0.2 & 1.5 & 2.5247 & 1.83 & 7.029 & 2.702 & 68.744 & 1.22 & 17.186 & 0.2 \\
\hline 2f & 4 & 0.5 & 1.5 & 2.5314 & 1.327 & 6.402 & 2.702 & 68.7209 & 0.8846 & 17.1802 & 0.2 \\
\hline 3a & 3 & 0.2 & 2 & 3.0635 & 2.219 & 10.369 & 4.6429 & 68.644 & 1.1095 & 22.8813 & 0.15 \\
\hline 3b & 3 & 0.5 & 2 & 3.0426 & 1.81 & 9.7552 & 4.6429 & 68.885 & 0.905 & 22.9616 & 0.15 \\
\hline 3c & 3.5 & 0.2 & 2 & 3.2339 & 2.149 & 11.3542 & 4.6429 & 68.644 & 1.0745 & 19.6125 & 0.175 \\
\hline 3d & 3.5 & 0.5 & 2 & 3.2364 & 2.017 & 10.338 & 4.6429 & 68.72 & 1.0085 & 19.6342 & 0.175 \\
\hline 3e & 4 & 0.2 & 2 & 3.3723 & 2.368 & 12.1038 & 4.6429 & 68.814 & 1.184 & 17.2035 & 0.2 \\
\hline 3f & 4 & 0.5 & 2 & 3.3802 & 2.427 & 11.2299 & 4.6429 & 68.850 & 1.2135 & 17.2125 & 0.2 \\
\hline
\end{tabular}

For position C
\begin{tabular}{|l|l|l|l|l|l|l|l|l|l|l|l|}
\hline GROYNE & $\begin{array}{l}\text { Length } \\
\text { of } \\
\text { groyne } \\
(\mathrm{m})\end{array}$ & $\begin{array}{l}\text { Width } \\
\text { of } \\
\text { groyne } \\
(\mathrm{m})\end{array}$ & $\begin{array}{l}\text { Approach } \\
\text { velocity } \\
\mathrm{V}_{\text {app }} \\
(\mathrm{m} / \mathrm{s})\end{array}$ & $\begin{array}{l}\text { Maximum } \\
\text { velocity } \\
\mathrm{V}_{\max } \\
(\mathrm{m} / \mathrm{s})\end{array}$ & $\begin{array}{l}\text { Tip } \\
\text { velocity } \\
\mathrm{V}_{\text {tip }} \\
(\mathrm{m} / \mathrm{s})\end{array}$ & $\begin{array}{l}\text { Max. } \\
\text { shear } \\
\text { stress, } \\
\Gamma_{\text {max }} \\
(\mathrm{Pa})\end{array}$ & $\begin{array}{l}\text { Initial } \\
\text { shear } \\
\text { stress, } \\
\Gamma_{\mathrm{o}} \\
(\mathrm{Pa})\end{array}$ & $\begin{array}{l}\text { Protected } \\
\text { length, D } \\
(\mathrm{m})\end{array}$ & $\mathrm{V}_{\text {tip }} / \mathrm{V}_{\text {app }}$ & $\mathrm{D} / \mathrm{L}$ & $\mathrm{L} / \mathrm{B}$ \\
\hline 1a & 3 & 0.2 & 1 & 1.2935 & 1.0588 & 1.8231 & 1.259 & 49.804 & 1.0588 & 16.601 & 0.15 \\
\hline 1b & 3 & 0.5 & 1 & 1.2996 & 1.058 & 1.6718 & 1.259 & 49.895 & 1.058 & 16.631 & 0.15 \\
\hline 1c & 3.5 & 0.2 & 1 & 1.3301 & 1.074 & 1.8805 & 1.259 & 49.725 & 1.074 & 14.207 & 0.175 \\
\hline $1 \mathrm{~d}$ & 3.5 & 0.5 & 1 & 1.3326 & 1.07 & 1.78135 & 1.259 & 49.829 & 1.07 & 14.236 & 0.175 \\
\hline 1e & 4 & 0.2 & 1 & 1.655 & 1.116 & 2.0346 & 1.259 & 49.7711 & 1.116 & 12.4427 & 0.2 \\
\hline $1 \mathrm{f}$ & 4 & 0.5 & 1 & 1.3751 & 1.120 & 1.8912 & 1.259 & 49.91 & 1.12 & 12.4775 & 0.2 \\
\hline 2a & 3 & 0.2 & 1.5 & 1.941 & 1.564 & 3.9299 & 2.702 & 49.68 & 1.0426 & 16.56 & 0.15 \\
\hline 2b & 3 & 0.5 & 1.5 & 1.9515 & 1.4947 & 3.595 & 2.702 & 49.853 & 0.9964 & 16.617 & 0.15 \\
\hline 2c & 3.5 & 0.2 & 1.5 & 1.9955 & 1.592 & 4.067 & 2.702 & 49.61 & 1.0613 & 14.1742 & 0.175 \\
\hline 2d & 3.5 & 0.5 & 1.5 & 2.0007 & 1.5813 & 3.858 & 2.702 & 49.589 & 1.0542 & 14.1682 & 0.175 \\
\hline
\end{tabular}




\begin{tabular}{|l|l|l|l|l|l|l|l|l|l|l|l|}
\hline 2e & 4 & 0.2 & 1.5 & 2.0492 & 1.616 & 4.3486 & 2.702 & 49.844 & 1.0773 & 12.461 & 0.2 \\
\hline 2f & 4 & 0.5 & 1.5 & 2.0699 & 1.5066 & 3.980 & 2.702 & 49.938 & 1.0044 & 12.4845 & 0.2 \\
\hline 3a & 3 & 0.2 & 2 & 2.590 & 2.114 & 6.7209 & 4.6429 & 49.598 & 1.057 & 16.5326 & 0.15 \\
\hline 3b & 3 & 0.5 & 2 & 2.6048 & 1.951 & 6.037 & 4.6429 & 49.898 & 0.9755 & 16.632 & 0.15 \\
\hline 3c & 3.5 & 0.2 & 2 & 2.6637 & 2.079 & 6.9739 & 4.6429 & 49.58 & 1.0395 & 14.1657 & 0.175 \\
\hline 3d & 3.5 & 0.5 & 2 & 2.6747 & 2.157 & 6.374 & 4.6429 & 49.78 & 1.0785 & 14.2228 & 0.175 \\
\hline 3e & 4 & 0.2 & 2 & 2.736 & 2.2281 & 7.3845 & 4.6429 & 49.843 & 1.11405 & 12.4607 & 0.2 \\
\hline 3f & 4 & 0.5 & 2 & 2.7670 & 2.187 & 6.829 & 4.6429 & 49.619 & 1.0935 & 12.4047 & 0.2 \\
\hline
\end{tabular}

Now having the most efficient size of groyne on the basis of tip velocity and shear stress, the groyne is then installed at different angles w.r.t. the horizontal. The following table gives the values of tip velocity, maximum velocity and protected length at different positions with different velocities.

For position A

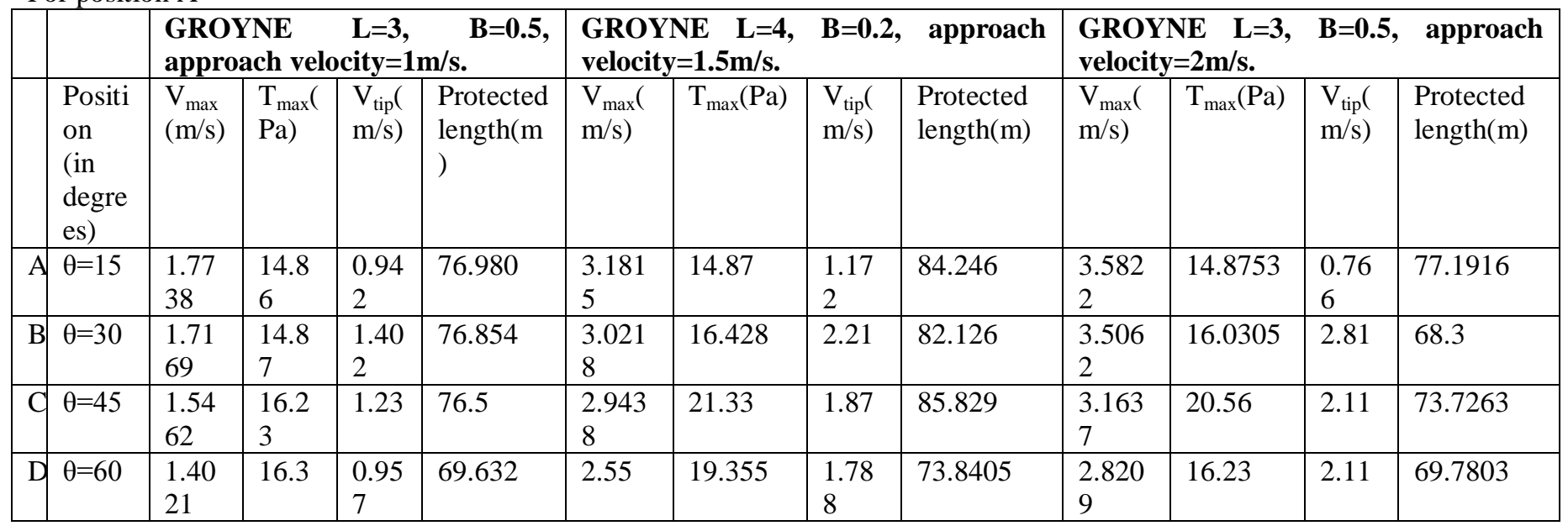

\begin{tabular}{|c|c|c|c|c|c|c|c|c|c|c|c|c|c|}
\hline \multicolumn{14}{|c|}{ For position $\mathrm{B}$} \\
\hline & & $\begin{array}{l}\text { GRO } \\
\text { appr }\end{array}$ & $\begin{array}{l}\mathrm{NE} \\
\text { ch vel }\end{array}$ & $\begin{array}{l}\mathrm{L}=3 \text {, } \\
\text { ity }=1\end{array}$ & S. & $\begin{array}{l}\text { GRO } \\
\text { veloci }\end{array}$ & $\begin{array}{l}\mathrm{VE} \quad \mathrm{L}=3, \\
=1.5 \mathrm{~m} / \mathrm{s} .\end{array}$ & $B=0.5$ & approach & $\begin{array}{l}\text { GROYI } \\
\text { velocity }\end{array}$ & $\begin{array}{l}\mathbf{E} \quad \mathbf{L}=3, \\
2 \mathrm{~m} / \mathrm{s} .\end{array}$ & $\mathrm{B}=\mathbf{0}$. & approach \\
\hline & $\begin{array}{l}\text { Positi } \\
\text { on } \\
\text { (in } \\
\text { degre } \\
\text { es) }\end{array}$ & $\begin{array}{l}\mathrm{V}_{\max } \\
(\mathrm{m} / \mathrm{s} \\
)\end{array}$ & $\begin{array}{l}\mathrm{T}_{\max }( \\
\mathrm{Pa})\end{array}$ & $\begin{array}{l}V_{\text {tip }} \\
(\mathrm{m} / \\
\mathrm{s})\end{array}$ & $\begin{array}{l}\text { Protected } \\
\text { length }(\mathrm{m} \\
)\end{array}$ & $\begin{array}{l}V_{\max }( \\
\mathrm{m} / \mathrm{s})\end{array}$ & $\mathrm{T}_{\max }(\mathrm{Pa})$ & $\begin{array}{l}V_{\text {tip }}( \\
\mathrm{m} / \mathrm{s})\end{array}$ & $\begin{array}{l}\text { Protected } \\
\text { length }(\mathrm{m})\end{array}$ & $\begin{array}{l}\mathrm{V}_{\max }(\mathrm{m} \\
/ \mathrm{s})\end{array}$ & $\mathrm{T}_{\max }(\mathrm{Pa})$ & $\begin{array}{l}V_{\text {tip }}( \\
m / s)\end{array}$ & $\begin{array}{l}\text { Protected } \\
\text { length }(m)\end{array}$ \\
\hline A & $\theta=15$ & $\begin{array}{l}1.56 \\
67\end{array}$ & 2.80 & $\begin{array}{l}0.0 \\
044\end{array}$ & 78.411 & 2.532 & 7.2202 & $\begin{array}{l}1.60 \\
91\end{array}$ & 75.864 & 3.204 & 10.999 & $\begin{array}{l}0.95 \\
63\end{array}$ & 73.9806 \\
\hline B & $\theta=30$ & $\begin{array}{l}1.58 \\
71\end{array}$ & $\begin{array}{l}3.585 \\
7\end{array}$ & $\begin{array}{l}1.0 \\
86\end{array}$ & 72.248 & $\begin{array}{l}2.580 \\
5\end{array}$ & 9.0326 & $\begin{array}{l}1.65 \\
7\end{array}$ & 74 & 3.2413 & 13.359 & $\begin{array}{l}1.08 \\
85\end{array}$ & 54 \\
\hline C & $\theta=45$ & $\begin{array}{l}1.55 \\
48\end{array}$ & $\begin{array}{l}3.798 \\
7\end{array}$ & $\begin{array}{l}0.0 \\
123\end{array}$ & 73.1683 & $\begin{array}{l}2.504 \\
9\end{array}$ & 9.5156 & $\begin{array}{l}1.73 \\
0\end{array}$ & 75.2085 & 3.1675 & 14.215 & $\begin{array}{l}1.65 \\
6\end{array}$ & 73.69 \\
\hline & $\theta=60$ & $\begin{array}{l}1.47 \\
32\end{array}$ & $\begin{array}{l}3.999 \\
0\end{array}$ & $\begin{array}{l}0.9 \\
814\end{array}$ & 72.5419 & 2.368 & 9.1283 & $\begin{array}{l}1.50 \\
77\end{array}$ & 73.988 & 3.015 & 14.9604 & $\begin{array}{l}1.49 \\
6\end{array}$ & 72.039 \\
\hline
\end{tabular}

\begin{tabular}{|c|c|c|c|c|c|c|c|c|c|c|c|c|}
\hline \multicolumn{13}{|c|}{ in $\mathrm{C}$} \\
\hline & \multicolumn{4}{|c|}{$\begin{array}{l}\text { GROYNE } \quad \mathrm{L}=3, \quad \mathrm{~B}=\mathbf{0 . 5}, \\
\text { approach velocity=1m/s. }\end{array}$} & \multicolumn{4}{|c|}{$\begin{array}{l}\text { GROYNE } \mathrm{L}=3, \quad B=0.5, \text { approach } \\
\text { velocity }=1.5 \mathrm{~m} / \mathrm{s} \text {. }\end{array}$} & \multicolumn{4}{|c|}{$\begin{array}{l}\text { GROYNE } \quad \mathrm{L}=3, \quad \mathrm{~B}=0.5, \quad \text { approach } \\
\text { velocity }=2 \mathrm{~m} / \mathrm{s} \text {. }\end{array}$} \\
\hline $\begin{array}{l}\text { Positi } \\
\text { on } \\
\text { (in } \\
\text { degre } \\
\text { es) }\end{array}$ & $\begin{array}{l}\mathrm{V}_{\max } \\
(\mathrm{m} / \mathrm{s} \\
)\end{array}$ & $\begin{array}{l}T_{\max }( \\
\mathrm{Pa})\end{array}$ & $\begin{array}{l}\mathrm{V}_{\text {tip }} \\
(\mathrm{m} / \\
\mathrm{s})\end{array}$ & $\begin{array}{l}\text { Protected } \\
\text { length }(\mathrm{m} \\
)\end{array}$ & $\begin{array}{l}V_{\max }( \\
m / s)\end{array}$ & $\mathrm{T}_{\max }(\mathrm{Pa})$ & $\begin{array}{l}\mathrm{V}_{\text {tip }}( \\
\mathrm{m} / \mathrm{s})\end{array}$ & $\begin{array}{l}\text { Protected } \\
\text { length }(\mathrm{m})\end{array}$ & $\begin{array}{l}\mathrm{V}_{\max }(\mathrm{m} \\
/ \mathrm{s})\end{array}$ & $\mathrm{T}_{\max }(\mathrm{Pa})$ & $\begin{array}{l}\mathrm{V}_{\text {tip }}( \\
\mathrm{m} / \mathrm{s})\end{array}$ & $\begin{array}{l}\text { Protected } \\
\text { length }(\mathrm{m})\end{array}$ \\
\hline
\end{tabular}




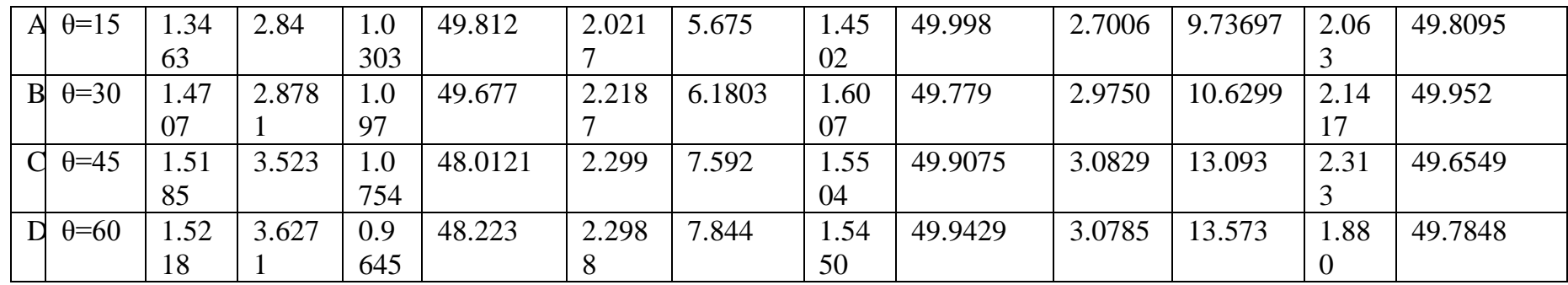

\section{Results and Discussions:-}

Variation of factor $\mathrm{d} / \mathrm{l}$ with factor $\mathrm{l} / \mathrm{b}$ for different positions of the groyne.

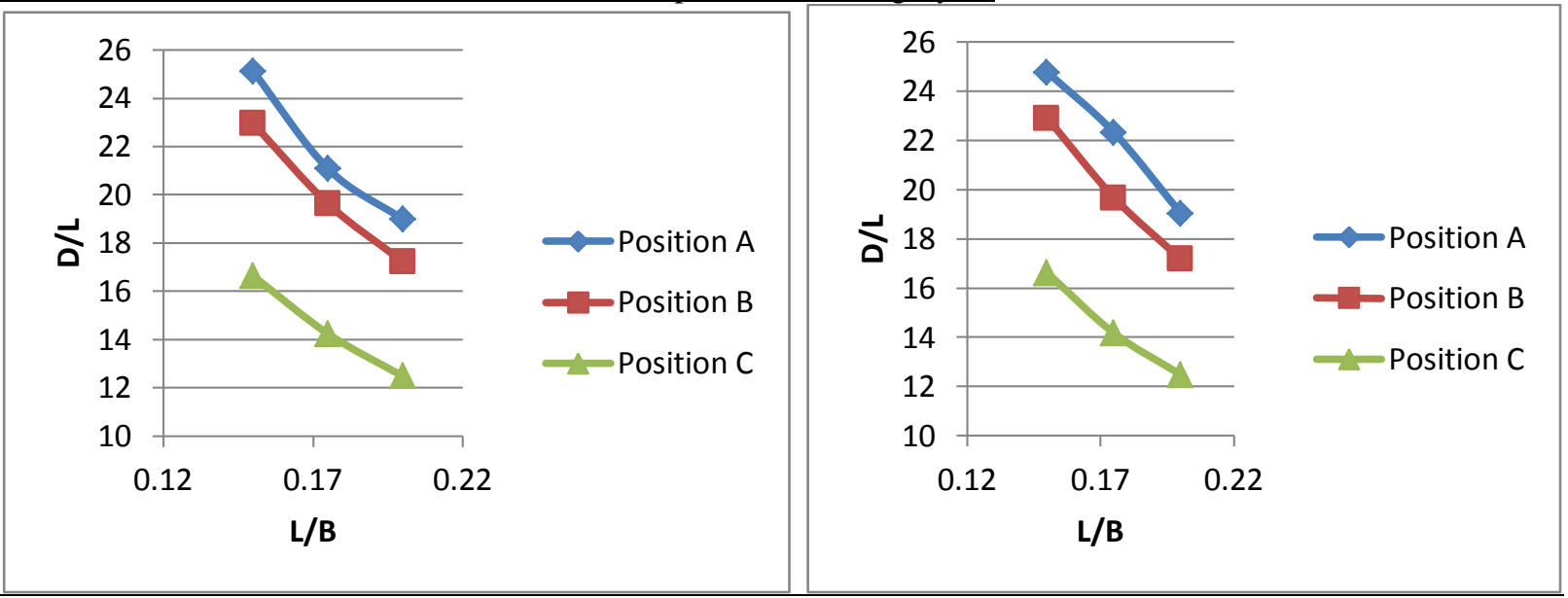

Fig. 3:- Variation of $\mathrm{D} / \mathrm{L}$ with $\mathrm{L} / \mathrm{B}$ for $\mathrm{V}=1 \mathrm{~m} / \mathrm{s}$

Fig.4:- Variation of $\mathrm{D} / \mathrm{L}$ with $\mathrm{L} / \mathrm{B}$ for $\mathrm{V}=1.5 \mathrm{~m} / \mathrm{s}$

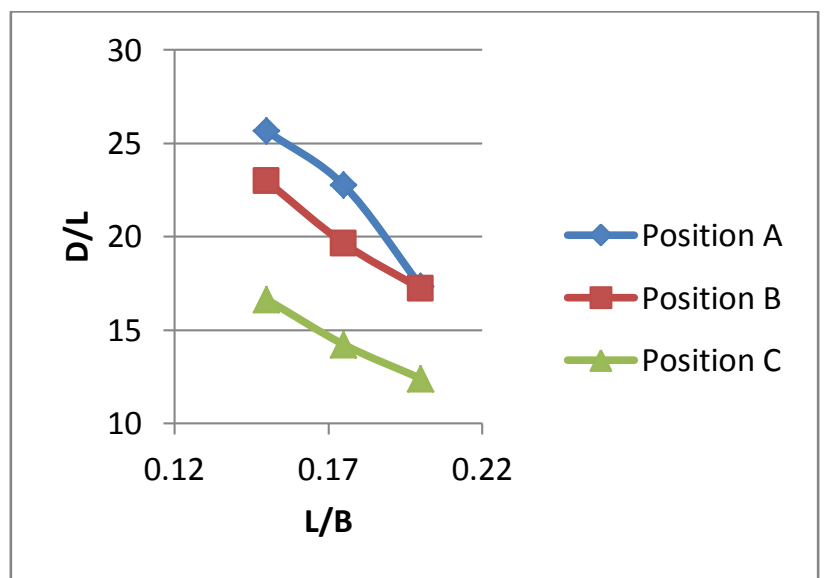

Fig.5:- Variation of D/L with L/B for Velocity $2 \mathrm{~m} / \mathrm{s}$

It was observed that the variation was not linear, as expected there are other factors that influence the Protection Length. It can be inferred from the variation that with increase in $\mathrm{L} / \mathrm{B}$ ratio the value of $\mathrm{D} / \mathrm{L}$ decreases for almost all the cases of placement of groyne. Protection length is maximum for position A for all the groyne sizes at all velocities and a good protection length is obtained for position $\mathrm{B}$. 
Variation of factor $1 / b$ with factor $v_{\text {tip }} / v_{\text {app }}$ for different positions of the groyne:-

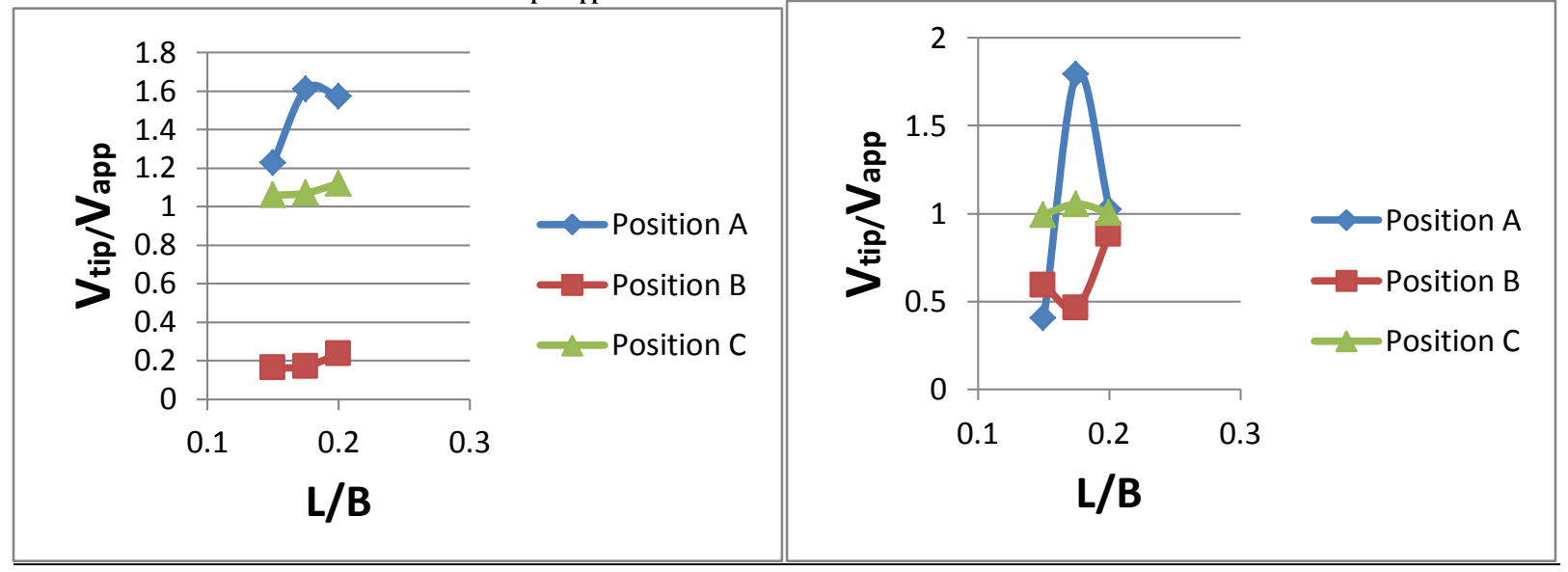

Fig.6:- Variation of $\mathrm{V}_{\text {tip }} / \mathrm{V}_{\text {app }}$ with $\mathrm{L} / \mathrm{B}$ for $\mathrm{V}=1 \mathrm{~m} / \mathrm{s}$.

Fig.7:- Variation of $\mathrm{V}_{\text {tip }} / \mathrm{V}_{\text {app }}$ with $\mathrm{L} / \mathrm{B}$ for $\mathrm{V}=1.5 \mathrm{~m} / \mathrm{s}$.

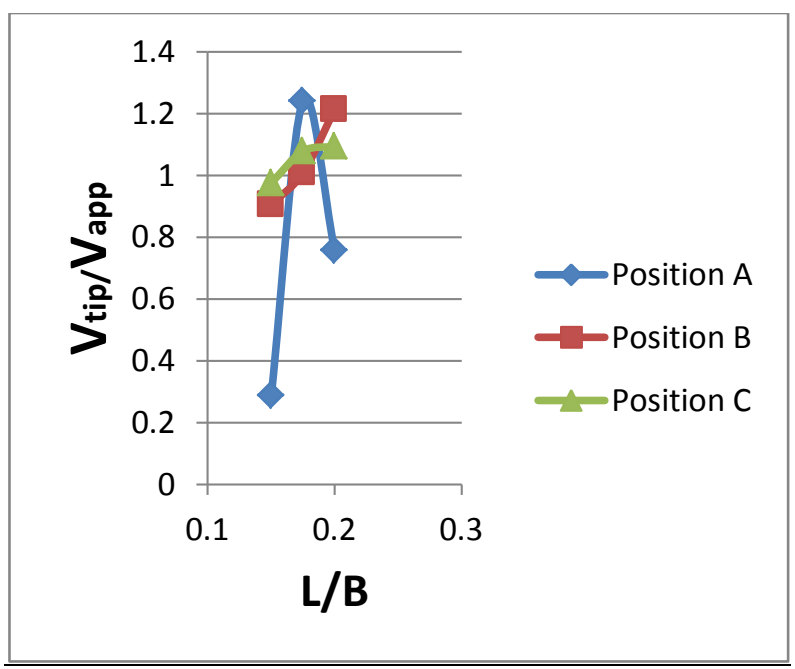

Fig.8:- Variation of $\mathrm{V}_{\text {tip }} / \mathrm{V}_{\text {app }}$ with $\mathrm{L} / \mathrm{B}$ for $\mathrm{V}=2 \mathrm{~m} / \mathrm{s}$.

It can be concluded from the variation that with increase in $\mathrm{L} / \mathrm{B}$ ratio the value of $\mathrm{V}_{\text {tip }} / \mathrm{V}_{\text {app }}$ increases for more of the cases of placement of groyne at different positions and the variation is found to be non-linear, so it was expected that there are other factors that influence the tip velocity.

The position that should be noted is position A, for a $\mathrm{L} / \mathrm{B}$ ratio of 0.175 , that is Length of groyne $=3.5 \mathrm{~m}$. At this position for all the velocities of flow it can be seen that there is sudden drop in the $\mathrm{V}_{\text {tip }} / \mathrm{V}_{\text {app }}$ factor or the tip velocity. The tip velocity and the maximum velocity are found near the groyne and thus it contributes to the formation of vortices that lead to scouring. Owing to the curvature of the section it can be seen that the velocities here also contribute to the production of centrifugal forces on the bend which in turn will cause more scouring. Free vortex is formed where the curvature is more and where the tip velocity is maximum thus creating more disturbances as that might be observed in a straight channel.

Variation of Factor $T_{\max }$ For Different Groyne Position:-

Analysis of the variation shows that the maximum shear stress increases with increase in installation angle of groyne for almost all the velocities. Thus a maximum value of $21.33 \mathrm{~Pa}$ for velocity $1.5 \mathrm{~m} / \mathrm{s}$ was observed for groyne size of $3 \mathrm{~m}$ at position $\mathrm{A}$ and minimum shear stress of $2.80 \mathrm{~Pa}$ for velocity of $1 \mathrm{~m} / \mathrm{s}$ at position $\mathrm{B}$. It can also be seen that in each case for various velocities the minimum value occurs at position ' $a$ '. This shows that this position is the most efficient i.e. at angle $\theta=15^{\circ}$ among all the position for all velocities. 


\section{Conclusions:-}

According to the case of groyne placement to the river of radius bend $=40 \mathrm{~m}$, the groyne in $1 / 4$ upstream arc curves bend give a better effect to control the flow than another groyne placement. The position 'a' of groyne that is placed at an angle of $15^{\circ}$ with the horizontal on $1 / 4^{\text {th }}$ upstream arc is the most efficient position as the maximum shear stress is found to be minimum at this position, tip velocity at the groyne is also relatively very low and Protected length is also good at different approach velocities considered. Thus on the basis of the above studies it is the best placement of groyne to pass the flow through the channel bend safely and protect the concave face from erosion.

Suggestion for the groyne size can be also given based on the trend seen in the various factors during the iterations. The factors maximum shear stress, tip velocity and protected length are found to be in appreciable range for L/B ratio of 0.175 that is groyne size of $3.5 \mathrm{~m}$ and width $0.5 \mathrm{~m}$.

\section{References:-}

1. Suharjoko (1999) Study on Numerical Modeling of Two-Dimensional Horizontal Flow special case Groyne on the River Estuary, Thesis for the degree of Master Science in Civil Engineering Program, Department of Engineering Science, Post-graduate Program, University Of Gajah Mada, Yogyakatra.

2. Suharjoko (2001) Numerical Modeling of Two-Dimensional Horizontal Flow on Groyne Field due to Groyne Placement on the River Straight, Researches Report, Department of Research and Applications, Institute of Technology Sepuluh Nopember, Surabaya.

3. Jungseok Ho, Hong Koo Yeo, Julie Coonrod, and Won-Sik Ahn (2005) NUMERICAL MODELING STUDY FOR FLOW PATTERN CHANGES INDUCED BY SINGLE GROYNE, Seminario Internacional La Hidroinformática en la Gestión Integrada de los Recursos Hídricos, Universidad del Valle/Instituto Cinara Kutija, V. and Murray, M. G. 162.

4. Kashyap, Shalini (2010) NUMERICAL MODELING OF FLOW AROUND SUBMERGED GROYNES IN A SHARP BEND USING LARGE EDDY SIMULATION. Diss. University of Ottawa.

5. FLUENT user's guide manual-version 6.3.26. Fluent Incor.

6. "Numerical Simulation of the Angle of Groyne Installation on the Separation Zone Length Behind it", by Hamid Shamloo and Bahareh Pirzadeh, Associate Professor at K.N. Toosi University of Technology.

7. Krishna Prasad, S. Indulekha, Balan. K (2015) Analysis of groyne placement on minimizing river bank protection, College of Engineering Trivandram.

8. J. Yajdi, H. Sarkardeh, Aminuddin N. Ghani (2010) 3D Simulation flow around a single spur dike with freesurface flow, Water Research Institute.

9. Suharjoko, Mohammad Bisri, Rispiningtati, Muhammad Ruslin Anwar, "An Analysis Of The Groyne Placement At The River Bend Based On Current Flow Be Occurred”, Brawijaya University, Malang.

10. Mohammad Vaghefi, Masoud Ghodsian, Maryam Akbari (2016) Experimental Investigation on 3D Flow around a Single T-Shaped Spur Dike in a Bend, Persian Gulf University. 